\title{
Comparación de la densidad capilar parafoveal entre sujetos sanos y diabéticos con y sin retinopatía
}

\section{Virgilio Lima-Gómez *, Dulce Milagros Razo Blanco-Hernández² y Selma Alin Somilleda-Ventura}

${ }^{1}$ Servicio de Oftalmología; ${ }^{2}$ División de Investigación. Hospital Juárez de México, Ciudad de México; ${ }^{3}$ Dirección de Investigación y Enseñanza, Hospital Juárez de México, Ciudad de México, México.

\section{Resumen}

Antecedentes: Los cambios de densidad parafoveal no se han caracterizado en diabéticos tipo 2 sin retinopatía. Objetivo: Comparar la densidad capilar parafoveal entre sujetos sanos y diabéticos con y sin retinopatía. Método: Estudio observacional, prospectivo, transversal y comparativo. Se incluyeron sujetos sin diabetes (grupo 1), diabéticos tipo 2 sin y con retinopatía (grupos 2 y 3), y con edema macular (grupo 4), sin isquemia macular. La variable de estudio fue la densidad capilar parafoveal, y las variables predictoras fueron las mediciones de la ZAF, el grosor retiniano y el grupo. Se compararon las diferencias entre grupos mediante las pruebas de Kruskal-Wallis y Rho de Spearman. Resultados: 144 ojos; la densidad capilar parafoveal del grupo 1 superó la de los restantes $(p<0.05)$. El área, el perímetro y el diámetro de la ZAF fueron mayores en el grupo 3. Existió correlación positiva entre la densidad capilar parafoveal y el grosor del campo central en los grupos 1, 2, y 3. Conclusiones: La densidad capilar parafoveal disminuye conforme avanza el daño por diabetes tipo 2; puede existir una reducción en sujetos con diabetes sin retinopatía, con grosor retiniano y ZAF normales. El impacto clínico de este hallazgo requiere evaluación adicional.

PALABRAS CLAVE: Densidad capilar parafoveal. Diabetes. Retinopatía diabética. Tomografía de coherencia óptica. Zona avascular foveal.

\section{Abstract}

Background: Parafoveal capillary density changes have not been characterized in type 2 diabetics without retinopathy. Objective: To compare parafoveal capillary density between healthy subjects, and diabetics with and without retinopathy. Method: Observational, prospective, transversal and comparative study; subjects without diabetes (group 1), diabetics without retinopathy (group 2), with retinopathy (group 3) and with diabetic macular edema (group 4), without macular ischemia, were included. The study variable was parafoveal capillary density, and the predictor variables were the measures of the foveal avascular zone, retinal thickness and the group. The variables were compared between groups using Kruskal-Wallis and Spearman's Rho tests. Results: One hundred and forty eyes were evaluated; parafoveal capillary density was higher in group 1 than in the rest ( $p<0.05)$. Area, perimeter and diameter of the foveal avascular zone were higher in group 3. A positive correlation existed a positive between parafoveal capillary density and central field thickness in groups 1,2 and 3. Conclusion: Parafoveal capillary density decreases as diabetes-induced damage increase; a reduction may exist in diabetics without retinopathy and normal retinal thickness and foveal avascular zone are normal. The clinical impact of this finding requires further evaluation.

KEY WORDS: Parafoveal capillary density. Diabetes. Diabetic retinopathy. Optical coherence tomography. Foveal avascular zone.

\author{
Correspondencia: \\ *Virgilio Lima-Gómez \\ Av. Instituto Politécnico Nacional, 5160 \\ Col. Magdalena de las Salinas, Del. Gustavo A. Madero \\ C.P. 06770, Ciudad de México, México \\ E-mail: forscher7@gmail.com
}

Fecha de recepción: 16-08-2018

Fecha de aceptación: 20-08-2018 DOI: 10.24875/GMM. 18004575
Gaceta Med Mex. 2018;154(Suppl 2): S30-S35

Disponible en PubMed www.gacetamedicademexico.com 


\section{Introducción}

La red capilar parafoveal delimita una región de la retina desprovista de vasos, concéntrica a la fóvea, conocida como zona avascular foveal (ZAF). ${ }^{1}$ Mediante angiografía con fluoresceína, su diámetro en los sujetos normales es de $0.88 \pm 0.16 \mathrm{~mm},{ }^{2}$ y aumenta cuando se ocluyen los capilares parafoveales; este aumento de la ZAF indica isquemia en la mácula, que puede presentarse en ojos con retinopatía diabética ${ }^{3}$ y se asocia con disminución de la agudeza visual. ${ }^{4}$

El análisis de los capilares parafoveales mediante angiografía con tomografía de coherencia óptica, que define el trayecto vascular con base en el flujo eritrocitario, ${ }^{5}$ ha detectado dimensiones de ZAF congruentes con las reportadas mediante fluorangiografía. ${ }^{6} \mathrm{En}$ los sujetos sanos, la ZAF puede variar en área y forma sin que se afecte la agudeza visual, ${ }^{7}$ y se ha descrito una correlación negativa entre el diámetro de la ZAF y el grosor foveal central $(r=-0.80 ; p<0.0001) .{ }^{8}$ La ZAF aumenta en los sujetos con retinopatía diabética no proliferativa, sin isquemia macular. ${ }^{9}$

Otra variable que mide la angiografía por tomografía de coherencia óptica es la densidad de capilares parafoveales, que disminuye en etapas tempranas de la retinopatía diabética, aunque no aumente la ZAF ni disminuya la agudeza visual. ${ }^{10}$ La densidad de capilares parafoveales baja conforme aumenta la gravedad de la retinopatía diabética $(r=-0.72$ para el plexo capilar superficial). ${ }^{3}$ Simonett, et al. ${ }^{11}$ identificaron una tendencia $(p=0.52)$ hacia una menor densidad capilar parafoveal en diabéticos tipo 1, al compararlos con sujetos sin diabetes. Cao, et al. ${ }^{12}$ reportaron una reducción de la densidad capilar parafoveal en sujetos diabéticos tipo 2 sin retinopatía, pero el $25 \%$ de sus casos presentaron falta de perfusión capilar foveal.

La sensibilidad retiniana (una variable funcional) puede disminuir en los sujetos diabéticos antes de que exista retinopatía o disminuya la agudeza visual; ${ }^{13}$ esto podría deberse a una densidad reducida de capilares parafoveales que no fuera evidente, si el grosor del campo central y el área de la ZAF fueran normales. La diferencia entre sujetos sin diabetes y sujetos con diabetes sin retinopatía no se ha reportado. Se desarrolló un estudio para comparar la densidad de capilares parafoveales en sujetos sin diabetes con la de sujetos con diabetes, con y sin retinopatía diabética, en quienes no existieran características de isquemia macular.

\section{Método}

Se realizó un estudio observacional, prospectivo, transversal y comparativo en sujetos con y sin diabetes, de la Ciudad de México y su área metropolitana. La muestra se obtuvo de los sujetos que asistieron a evaluación oftalmológica en un hospital federal de referencia. El estudio se desarrolló del 8 de enero al 28 de febrero de 2018, y se apegó a los criterios de la Declaración de Helsinki.

Se incluyeron sujetos con y sin diabetes tipo 2 que acudieron a valoración del fondo del ojo entre el 8 y el 26 de enero de 2018, con edad entre 18 y 80 años años, de ambos sexos, que tuvieran medios transparentes para poder realizar un estudio de tomografía de coherencia óptica con angiografía, y que aceptaran participar en el estudio mediante consentimiento bajo información por escrito. No se incluyeron los sujetos con uveítis, que tuvieran cualquier enfermedad retiniana distinta a retinopatía diabética ni que recibieran tratamiento con diuréticos o glucocorticoides por cualquier vía, y tampoco los que tenían fotocoagulación; se eliminaron los sujetos que retiraron su consentimiento bajo información y los que tuvieron un área de la ZAF sugestiva de isquemia.

Un solo investigador obtuvo una fotografía del fondo del ojo (CR2plus AF, Canon, Irvine, CA) en todos los sujetos. Posteriormente, otro investigador obtuvo un cubo macular y un mapa de densidad vascular de $3 \times 3 \mathrm{~mm}$ con el equipo Cirrus $5000 \mathrm{HD}$ Angioplex (Zeiss, Meditec, Dublin, CA). Un investigador más calificó el grado de retinopatía diabética en cada sujeto y registró si existía edema macular.

Los sujetos se asignaron a uno de cuatro grupos: 1) sin diabetes; 2) diabetes sin retinopatía; 3) con retinopatía diabética sin edema macular; y 4) con edema macular. Para los grupos 1 y 2 se incluyó un solo ojo por sujeto, que se seleccionó en forma aleatoria; para los sujetos de los grupos 3 y 4 se seleccionó un ojo en forma aleatoria cuando la retinopatía era simétrica, y en los casos de asimetría se seleccionaron ambos ojos, cada uno de los cuales se asignó al grupo correspondiente. Se consideró que un paciente presentaba isquemia macular cuando el área de la ZAF superaba por más de dos desviaciones estándar al promedio reportado en pacientes con retinopatía diabética no proliferativa $\left(0.62 \mathrm{~mm}^{2}\right){ }^{9}$

En cada caso se midieron el grosor retiniano en nueve campos del mapa macular y el volumen macular, así como la densidad capilar en el plexo superficial, y el área y el perímetro de la ZAF. La variable en estudio 
Tabla 1. Distribución de las variables angiográficas, por grupo

\begin{tabular}{|c|c|c|c|c|}
\hline Variables & $\begin{array}{l}\text { Sin diabetes* } \\
\quad(n=37)\end{array}$ & $\begin{array}{l}\text { Diabetes } \sin \\
\text { retinopatía* }(n=56)\end{array}$ & $\begin{array}{l}\text { Diabetes con } \\
\text { retinopatía* }(n=36)\end{array}$ & $\begin{array}{l}\text { Retinopatía más edema } \\
\text { macular }^{*}(n=15)\end{array}$ \\
\hline \multicolumn{5}{|l|}{ Densidad capilar } \\
\hline Región central $\left(\mathrm{mm}^{-1}\right)$ & $10.89 \pm 0.55$ & $8.71 \pm 0.45$ & $6.81 \pm 0.41$ & $6.67 \pm 0.66$ \\
\hline Región interna (mm-1) & $21.32 \pm 0.35$ & $19.57 \pm 0.41$ & $18.62 \pm 0.52$ & $16.93 \pm 0.81$ \\
\hline Región completa $\left(\mathrm{mm}^{-1}\right)$ & $20.15 \pm 0.35$ & $18.51 \pm 0.37$ & $17.29 \pm 0.48$ & $15.77 \pm 0.78$ \\
\hline \multicolumn{5}{|l|}{$Z A F$} \\
\hline Área $\left(\mathrm{mm}^{2}\right)$ & $0.26 \pm 0.02$ & $0.27 \pm 0.02$ & $0.35 \pm 0.02$ & $0.24 \pm 0.06$ \\
\hline Perímetro (mm) & $2.23 \pm 0.10$ & $2.38 \pm 0.07$ & $2.70 \pm 0.08$ & $2.07 \pm 0.38$ \\
\hline Diámetro (mm) & $0.71 \pm 0.03$ & $0.76 \pm 0.02$ & $0.86 \pm 0.03$ & $0.66 \pm 0.12$ \\
\hline Circularidad & $0.65 \pm 0.02$ & $0.59 \pm 0.02$ & $0.60 \pm 0.02$ & $0.61 \pm 0.05$ \\
\hline
\end{tabular}

fue la densidad de capilares parafoveales, y las variables predictoras fueron el grupo asignado, el área de la ZAF, el diámetro de la ZAF, el grosor retiniano en cada campo del mapa macular y el volumen macular.

Se compararon las medianas y los rangos intercuartílicos de la densidad de los capilares parafoveales mediante la prueba de Kruskal-Wallis; las comparaciones entre dos grupos se realizaron mediante la prueba $\mathrm{U}$ de Mann-Whitney. Para el análisis inferencial se calcularon el promedio y los intervalos de confianza del $95 \%$ de la densidad capilar parafoveal y el área de la ZAF en cada grupo. Se realizó un análisis de correlación entre la densidad de los capilares parafoveales y las variables restantes mediante la prueba Rho de Spearman. Se consideró significativo un valor de $p<0.05$. La información se almacenó y analizó con la versión 22 del programa SPSS para Windows.

\section{Resultados}

Se evaluaron 144 ojos de 81 pacientes con edades entre 18 y 80 años (promedio: 52.83 años; error estándar: \pm 1.13 ). El $63.2 \%$ eran del sexo femenino. Del total de la muestra, el $74.3 \%$ padecían diabetes, con un tiempo de evolución entre 0.1 y 25 años (10.04 \pm 0.79 ), y el $89.3 \%$ recibía tratamiento con hipoglucemiantes orales. La capacidad visual LogMAR fue de +0.6 a $0.0(+0.16 \pm+0.02)(20 / 25)$.

Treinta y siete ojos se asignaron al grupo 1, 56 ojos al grupo 2, 36 ojos al grupo 3 y 15 ojos al grupo 4 . Los promedios de las variables angiográficas se presentan en la Tabla 1. La densidad capilar retiniana del grupo 1 fue mayor que la de los grupos restantes, tanto en la región central $(p<0.05)$ (Figura 1) como en la región externa $(p<0.05)$ (Figura 2$)$ y en la región

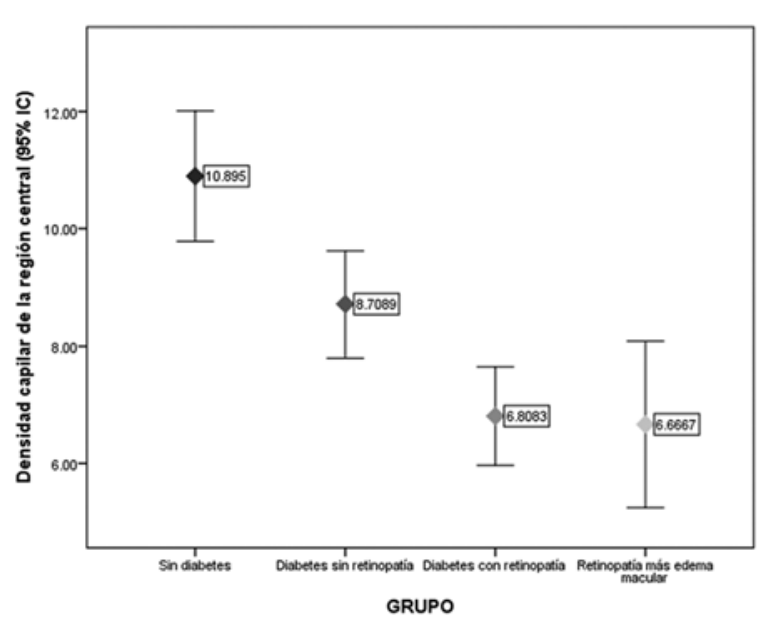

Figura 1. Comparación de la densidad capilar retiniana en la región central de la angiografía de $3 \times 3 \mathrm{~mm}$ entre grupos. IC: intervalo de confianza.

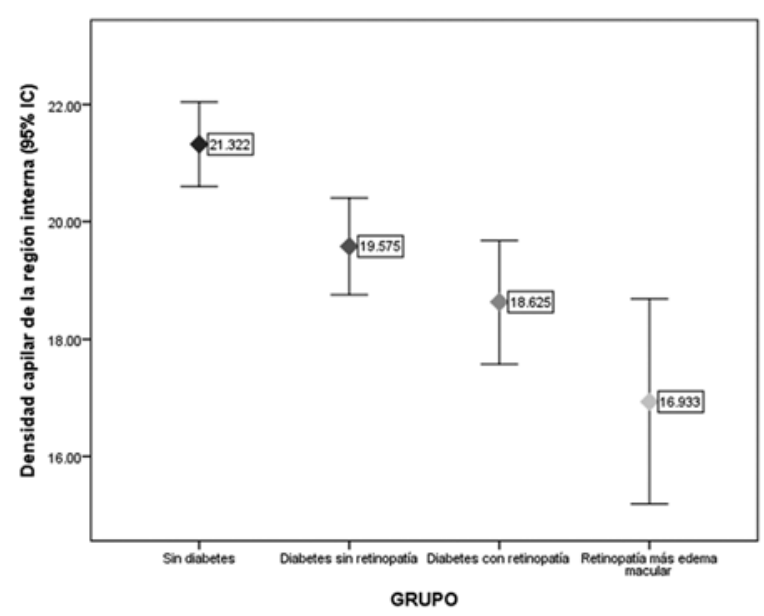

Figura 2. Comparación de la densidad capilar en la región interna de la angiografía $3 \times 3 \mathrm{~mm}$ entre grupos

el diámetro de la ZAF fueron mayores en el grupo 3 que en el resto de los grupos. 
Tabla 2. Distribución del grosor retiniano, por grupo

\begin{tabular}{lcccc}
\hline Variables $(\boldsymbol{\mu m})$ & $\begin{array}{c}\text { Sin diabetes* } \\
(\mathbf{n}=\mathbf{3 7})\end{array}$ & $\begin{array}{c}\text { Diabetes } \mathbf{s i n} \\
\text { retinopatía* }(\mathbf{n}=\mathbf{5 6})\end{array}$ & $\begin{array}{c}\text { Diabetes con } \\
\text { retinopatía* }(\mathbf{n}=\mathbf{3 6})\end{array}$ & $\begin{array}{c}\text { Retinopatía más edema } \\
\text { macular* }(\mathbf{n}=\mathbf{1 5})\end{array}$ \\
\hline Campo 1 $(\mathrm{GCC})$ & $249.70 \pm 3.64$ & $248.09 \pm 3.2$ & $243.94 \pm 3.69$ & $284.73 \pm 10.22$ \\
Campo 2 & $316.78 \pm 2.52$ & $316.66 \pm 3.11$ & $318.86 \pm 2.67$ & $335.47 \pm 6.34$ \\
Campo 3 & $303.70 \pm 2.30$ & $302.32 \pm 3.25$ & $303.30 \pm 3.18$ & $326.07 \pm 6.56$ \\
Campo 4 & $312.62 \pm 2.69$ & $310.98 \pm 2.86$ & $312.89 \pm 2.78$ & $329.47 \pm 5.71$ \\
Campo 5 & $316.59 \pm 2.67$ & $319.53 \pm 2.81$ & $317.80 \pm 3.00$ & $336.53 \pm 5.26$ \\
Campo 6 & $276.16 \pm 2.41$ & $276.96 \pm 1.93$ & $286.61 \pm 2.28$ & $308.07 \pm 6.21$ \\
Campo 7 & $260.67 \pm 2.13$ & $260.98 \pm 1.88$ & $270.44 \pm 2.38$ & $297.93 \pm 5.28$ \\
Campo 8 & $260.11 \pm 2.21$ & $262.23 \pm 1.99$ & $269.14 \pm 2.28$ & $288.80 \pm 2.25$ \\
Campo 9 & $288.16 \pm 2.89$ & $291.78 \pm 2.72$ & $298.47 \pm 2.23$ & $318.87 \pm 6.79$ \\
Volumen macular $\left(\mathrm{mm}^{3}\right)$ & $9.89 \pm 0.07$ & $9.92 \pm 0.07$ & $10.15 \pm 0.07$ & $10.94 \pm 0.14$
\end{tabular}

*Promedio \pm error estándar.

GCC: grosor del campo central

La distribución del grosor retiniano se presenta en la Tabla 2. El grosor en todos los campos y el volumen macular fueron mayores en el grupo 4 que en el 1; el grosor también fue mayor en el grupo 4 que en los grupos 2 y 3 ( $p<0.05)$, a excepción de los campos 2 y 4 . Adicionalmente, el grosor de los campos 6 $(p=0.003), 7(p=0.009), 8(p=0.01)$ y $9(p=0.01)$ del grupo 3 fue mayor que en el grupo 1 . El grosor del campo central no tuvo diferencia entre los grupos 1,2 y 3 , pero fue consistentemente mayor en el grupo 4 (Figura 3).

El grosor retiniano en los $3 \mathrm{~mm}$ centrales no difirió entre los grupos 1, 2 y 3, pero la densidad capilar retiniana disminuyó progresivamente del grupo 1 al 2, y del 2 al 3 . El grosor de los campos del anillo externo aumentó en el grupo 3 con respecto al grupo 1 $(p=0.032)$, pero no se evaluó la densidad capilar en esa región, ya que se localiza por fuera de $3 \mathrm{~mm}$ de diámetro; el volumen macular tuvo un aumento semejante al de los campos externos.

La densidad de capilares parafoveales disminuyó conforme aumentaba el daño por diabetes (según el grupo): región central $\mathrm{Rho}=-0.44$, región interna $\mathrm{Rho}$ -0.46 , y región completa Rho $=-0.48(p<0.05)$. Los promedios de las características de la ZAF aumentaron, pero solo se encontró correlación positiva entre el grupo y el área $(R h o=0.20)$ y entre el grupo y el perímetro (Rho $=0.27$ ). Se encontró una correlación positiva entre la densidad vascular de la región central y el grosor del campo central en los grupos 1, 2 y 3; en el grupo 3, esta correlación se extendió al

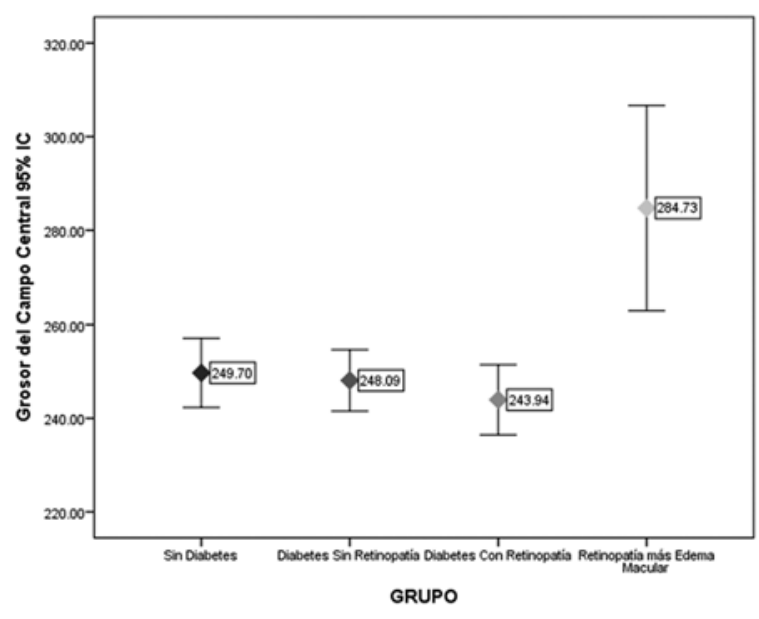

Figura 3. Comparación del grosor del campo central en micras, entre grupos

resto de los campos y al volumen macular (Tabla 3). El perímetro y el diámetro de la ZAF tuvieron una correlación negativa con el grosor del campo central en los grupos 1, 2 y 3 ; no se encontraron correlaciones significativas entre las variables vasculares y las de grosor macular en el grupo 4.

\section{Discusión}

La densidad capilar parafoveal en la región central disminuyó progresivamente conforme aumentó el daño por la diabetes, aunque el grosor de la mácula no se modificó; el grupo asignado tuvo correlación positiva con las variables de la ZAF, y negativa con las de la densidad capilar. 
Tabla 3. Correlaciones de las variables angiográficas y de grosor retiniano, entre grupos

\begin{tabular}{|c|c|c|c|c|}
\hline & Correlación & $\begin{array}{l}\text { Densidad capilar, } \\
\text { región central }\end{array}$ & Perímetro & Diámetro \\
\hline Sin diabetes & $\begin{array}{l}\text { Campo } 1 \text { (GCC) } \\
\text { Campo } 6\end{array}$ & $\begin{array}{c}0.49^{\dagger} \\
-0.41^{\star}\end{array}$ & $-0.47^{\dagger}$ & $-0.47^{\dagger}$ \\
\hline Diabetes sin retinopatía & Campo 1 (GCC) & $0.36^{+}$ & $-0.33^{\dagger}$ & $-0.33^{\dagger}$ \\
\hline Diabetes con retinopatía & $\begin{array}{l}\text { Campo } 1 \text { (GCC) } \\
\text { Campo } 2 \\
\text { Campo } 3 \\
\text { Campo } 4 \\
\text { Campo } 5 \\
\text { Campo } 6 \\
\text { Campo } 7 \\
\text { Campo } 8 \\
\text { Campo } 9 \\
\text { VM }\end{array}$ & $\begin{array}{l}0.54^{\dagger} \\
0.49^{\dagger} \\
0.55^{\dagger} \\
0.42^{\star} \\
0.53^{\dagger} \\
0.56^{\dagger} \\
0.42^{\star} \\
0.58^{\dagger} \\
0.64^{\dagger} \\
0.64^{\dagger}\end{array}$ & $-0.52^{\dagger}$ & $-0.52^{\dagger}$ \\
\hline
\end{tabular}

Retinopatía más edema macular

${ }^{*} p<0.01$.

$t p<0.05$

Prueba Rho de Spearman.

GCC: grosor del campo central; VM: volumen macular.

El promedio del área de la ZAF en este estudio fue menor que el reportado por $\mathrm{Di}$, et al. ${ }^{9}$ en sujetos $\sin$ diabetes y en sujetos con diabetes sin retinopatía. Los resultados concuerdan con los de esos autores ${ }^{9}$ en que la ZAF no varía significativamente entre sujetos con y sin diabetes. El mismo grupo ${ }^{9}$ también reportó que la ZAF aumentaba en sujetos con edema macular, comparada con aquellos que no lo tenían $(p=0.017)$, lo cual no se observó en nuestro estudio, probablemente porque no se incluyeron sujetos con áreas de la ZAF que sugirieran isquemia.

Chui, et al. ${ }^{8}$ reportaron que el área de la ZAF tiene una correlación negativa con el grosor del campo central $(r=-0.80 ; p<0.0001)$, pero no evaluaron la relación entre este y la densidad capilar. Ese fue el objetivo de nuestro estudio, en el que encontramos una correlación positiva del grosor del campo central con la densidad capilar (región central) en los grupos $1(r=0.49), 2(r=0.36)$ y $3(r=0.54)$, que se perdió en el grupo de edema macular diabético.

La diferencia de densidad de capilares parafoveales no se atribuyó a isquemia. Para evaluar el área de la ZAF se seleccionó el plexo superficial, ya que Ghassemi, et al. ${ }^{14}$ identificaron que en sujetos sanos es mayor que la del plexo profundo $\left(0.27 \pm 0.11 \mathrm{~mm}^{2} \mathrm{vs}\right.$. $0.35 \pm 0.12 \mathrm{~mm}^{2}$ ). Aun así, el área de la ZAF en ningún sujeto alcanzó una dimensión atípicamente alta como para considerarla sugestiva de isquemia macular.

La diferencia que reportaron Simonett, et al..$^{11}$ en diabéticos tipo 1, que no mostró significación estadística por el número reducido de ojos sin retinopatía
( $n=9$ ), se confirmó en este estudio en diabéticos tipo 2. Sería conveniente evaluar si enfermedades como la hipertensión arterial o la dislipidemia tienen una contribución significativa en la reducción de la densidad capilar parafoveal en sujetos que padecen diabetes tipo 2 y que no presentan retinopatía, como sugirió ese grupo. Los resultados de este estudio eliminan el potencial factor de confusión que representaba la falta de perfusión en los estudios de Cao, et al. ${ }^{12}$ y de Dimitrova, et al., ${ }^{15}$ y concuerdan en que el cambio en la densidad capilar parafoveal se presenta antes que los cambios clínicos de retinopatía diabética.

Los hallazgos de este estudio muestran que un área normal de la ZAF no indica que la densidad capilar parafoveal sea normal, y que la reducción de esta densidad es un cambio que puede presentarse en sujetos con diabetes antes de que se formen microaneurismas. Este cambio anatómico podría explicar las alteraciones funcionales que se han reportado en sujetos sin retinopatía diabética (como la reducción de la sensibilidad retiniana), y la respuesta variable al tratamiento del edema macular, en sujetos con semejante grosor retiniano y estado de las capas internas de la retina. ${ }^{16}$

Una potencial fortaleza de este estudio fue el recurrir a un equipo de angiografía por tomografía de coherencia óptica que no depende del operador, que ha sido utilizado en otros estudios que evalúan la densidad capilar retiniana y la ZAF. Una potencial limitación fue el no evaluar simultáneamente con fluorangiografía, que hubiera permitido identificar microaneurismas 
subclínicos y definir una relación de temporalidad con la disminución de la densidad de capilares parafoveales. Esta limitación podría haberse superado por la imagen de fondo de ojo obtenida (21 megapíxeles), que permite detectar cambios por debajo de la resolución oftalmoscópica.

El impacto clínico de la disminución temprana de la densidad de capilares parafoveales en sujetos con diabetes tipo 2 requiere evaluación adicional. Aunque es esperable que progrese conforme lo hace la retinopatía diabética, hasta el momento se desconoce si podría ser útil para tomar decisiones terapéuticas, tanto oculares como sistémicas.

\section{Conclusiones}

La disminución de la densidad de capilares parafoveales, que aumenta conforme avanza el daño por retinopatía diabética, se presentó también en sujetos con diabetes tipo 2 sin evidencia clínica de retinopatía, aun cuando su grosor retiniano y el área de su ZAF eran normales.

\section{Bibliografía}

1. Couturier A, Mane V, Bonnin S, Erginay A, Massin P, Gaudric A, et al. Capillary plexus anomalies in diabetic retinopathy on optical coherence tomography angiography. Retina. 2015;35:2384-91.

2. Wu LZ, Huang ZS, Wu DZ, Chan E. Characteristics of the capillary-free zone in the normal human macula. Jpn J Ophthalmol. 1985;29:406-11.

3. Kim AY, Chu Z, Shahidzadeh A, Wang RK, Puliafito CA, Kashani AH. Quantifying microvascular density and morphology in diabetic retinopathy using spectral-domain optical coherence tomography angiography. Invest Ophthalmol Vis Sci. 2016;57:362-70.

4. Freiberg FJ, Pfau M, Wons J, Wirth MA, Becker MD, Michels S. Optical coherence tomography angiography of the foveal avascular zone in diabetic retinopathy. Graefes Arch Clin Exp Ophthalmol. 2016;254:1051-8.

5. De Carlo TE, Baumal CR. Advances in optical coherence tomography angiography. US Ophthalmic Review. 2016;9:37-40.

6. Matsunaga D, Yi J, De Koo L, Ameri H, Puliafito CA, Kashani H. Optical coherence tomography angiography of diabetic retinopathy in human subjects. Ophthalmic Surg Lasers Imaging Retina. 2015;46:796-805.

7. Wang Q, Kocaoglu OP, Cense B, Bruestle J, Jonnal RS, Gao W, et al. Imaging retinal capillaries using ultrahigh-resolution optical coherence tomography and adaptive optics. Invest Ophthalmol Vis Sci. 2011;52:6292-9.

8. Chui TY, VanNasdale DA, Elsner AE, Burns SA. The association between the foveal avascular zone and retinal thickness. Invest Ophthalmol Vis Sci. 2014;55:6870-7.

9. Di G, Weihong Y, Xiao Z, Zhikun Y, Xuan Z, Yi Q, et al. A morphological study of the foveal avascular zone in patients with diabetes mellitus using optical coherence tomography angiography. Graefes Arch Clin Exp Ophthalmol. 2016;254:873-9.

10. De Carlo TE, Chin AT, Bonini Filho MA, Adhi M, Branchini L, Salz DA, et al. Detection of microvascular changes in eyes of patients with diabetes but not clinical diabetic retinopathy using optical coherence tomography angiography. Retina. 2015;35:2364-70.

11. Simonett JM, Scarinci F, Picconi F, Giorno P, De Geronimo D, Di Renzo A, et al. Early microvascular retinal changes in optical coherence tomography angiography in patients with type 1 diabetes mellitus. Acta Ophthalmol. 2017;95:e751-5.

12. Cao D, Yang D, Huang Z, Zeng Y, Wang J, Hu Y, et al. Optical coherence tomography angiography discerns preclinical diabetic retinopathy in eyes with type 2 diabetes without clinical diabetic retinopathy. Acta Diabetol. 2018;55:469-77.

13. Gella L, Raman R, Kulothungan V, Saumya Pal S, Ganesan S, Sharma T. Retinal sensitivity in subjects with type 2 diabetes mellitus: Sankara Nethralaya Diabetic Retinopathy Epidemiology and Molecular Genetics Study (SN-DREAMS II, Report No. 4). Br J Ophthalmol. 2016;100:808-13.

14. Ghassemi F, Mirshahi R, Bazvand F, Fadakar K, Faghihi H, Sabour S. The quantitative measurements of foveal avascular zone using optical coherence tomography angiography in normal volunteers. J Curr Ophthalmol. 2017:29:293-9.

15. Dimitrova G, Chihara E, Takahashi H, Amano H, Okazaki K. Quantitative retinal optical coherence tomography angiography in patients with diabetes without diabetic retinopathy. Invest Ophthalmol Vis Sci. 2017; 58:190-6.

16. Radwan SH, Soliman AZ, Tokarev J, Zhang L, van Kujik, Koozekanani DD. Association of disorganization of retinal inner layers with vision after resolution of center involved diabetic macular edema. JAMA Ophthalmol. 2015;133:820-5. 